\title{
Assessment of acute obstructive pancreatitis by magnetic resonance imaging: Predicting the occurrence of pancreatic fistula following pancreatoduodenectomy
}

\author{
ZHENSHAN SHI ${ }^{1}$, XIUMEI LI ${ }^{1}$, YUEMING LI ${ }^{1}$, RUIXIONG YOU ${ }^{1}$, DAIRONG CAO ${ }^{1}$, \\ QUNLIN CHEN $^{1}$, KAMISHA RAMEN ${ }^{2}$ and VIKASH SAHADEO LOOSA ${ }^{2}$ \\ ${ }^{1}$ Department of Radiology, First Affiliated Hospital of Fujian Medical University, Fuzhou, Fujian 350005; \\ ${ }^{2}$ Department of Radiology, Fujian Medical University, Fuzhou, Fujian 350001, P.R. China
}

Received June 21, 2018; Accepted October 20, 2018

DOI: $10.3892 /$ mco.2019.1799

\begin{abstract}
The aim of the present study was to assess the effect of preoperative acute obstructive pancreatitis on the risk of the occurrence of pancreatic fistula (PF) following pancreaticoduodenectomy. A total of 22 patients who developed postoperative PF were carefully matched with 22 control patients without PF according to demographic data, pancreatic pathology, presenting symptoms and other surgery-associated parameters. These parameters were compared between these two groups. The mean pancreatic apparent diffusion coefficient (ADC) values in the fistula group were $1.14 \pm 0.31 \times 10^{-3} \mathrm{~mm}^{2} / \mathrm{s}$, which was significantly decreased compared with the non-fistula group $\left(1.48 \pm 0.44 \times 10^{-3} \mathrm{~mm}^{2} / \mathrm{s}\right)$ $(\mathrm{P}=0.005)$. The pancreas-muscle signal intensity (SI) ratio on fat-suppressed T1 weighted image (rT1) in the fistula group was $1.71 \pm 0.25$, which was significantly increased compared with the non-fistula group $(1.25 \pm 0.29)(\mathrm{P}<0.001)$. The pancreas-muscle SI ratios on fat-suppressed T2 weighted image (T2WI) in the fistula group and the non-fistula group were $0.72 \pm 0.08$ and $0.62 \pm 0.07$, respectively $(\mathrm{P}=0.79)$. There was no significant difference in pancreas-muscle SI ratio on fat-suppressed T2-weighted image (rT2) value between these two groups. Based on the receiver operating characteristic curve, the optimal cut-off value of ADC as a criterion for prediction of pancreatic fistula was $1.29 \times 10^{-3} \mathrm{~mm}^{2} / \mathrm{s}$, which yielded a sensitivity of $77.3 \%$ and a specificity of $63.6 \%$. In conclusion, the severity of acute obstructive pancreatitis was negatively associated with ADC values and pancreas-muscle
\end{abstract}

Correspondence to: Dr Qunlin Chen, Department of Radiology, First Affiliated Hospital of Fujian Medical University, 20 Cha-Zhong Road, Fuzhou, Fujian 350005, P.R. China

E-mail:2375530372@qq.com

Key words: magnetic resonance imaging, pancreatitis, pancreatic fistula
SI ratio on rT1 images, which may be useful for predicting the occurrence of PF preoperatively.

\section{Introduction}

Pancreaticoduodenectomy (PD) is the most common and effective surgery for the treatment of pancreatic and periampullary carcinoma $(1,2)$. The evolution of surgical techniques and perioperative management had significantly decreased the postoperative complications and mortality in patients (3-5). Postoperative pancreatic fistula (PF) remains one of the most dangerous and severe complications following PD (6). Significant risk factors for increased PF have been demonstrated to be old age, soft pancreatic texture, a large pancreatic remnant volume, small pancreatic duct diameter, increased levels of pancreatic fat, pancreatic pathology and longer surgery times (7-9). Occlusion of the primary pancreatic duct by pancreatic head and periampullary tumors often leads to obstructive pancreatitis within a few weeks (10). The pathology of obstructive pancreatitis is characterized by pancreatic atrophy, fibrosis, and acute and chronic inflammatory infiltrate that may cause the morphological and functional destruction of the pancreas (11). Furthermore, previous studies have demonstrated that the pancreatic inflammatory environment may serve a critical role in pancreatic cancer progression (12). The magnetic resonance imaging (MRI) parameters include apparent diffusion coefficient (ADC) value and signal intensity on T1-weighted fat-suppressed images (rT1), which are used to quantitatively analyze differences between pancreatic cancer and pancreatitis $(9,13)$. The T2*-corrected Dixon technique and intravoxel incoherent motion (IVIM) diffusion-weighted (DW) imaging have been suggested to assess pancreatic steatosis and fibrosis and predict postoperative PF (8). However, at present, it remains unclear whether acute preoperative obstructive pancreatitis may be a risk factor for fistula development.

The purpose of the present study was designed to determine whether patients who have acute obstructive pancreatitis preoperatively are more likely to develop a PF compared with matched controls, and if so, to investigate whether the ADC value and signal intensity on rT1 images may be used as a preoperative predictive tool for fistula development. 


\section{Patients and methods}

Patients. The present retrospective study was approved by the Institutional Review Board of First Affiliated Hospital of Fujian Medical University (Fuzhou, China), and the requirement for informed consent was waived. The authors had no access to information that identified individual participants during or following data collection. From January 2010 to March 2016, a total of 124 patients (55 women and 69 men; mean age, 53 years) underwent PD, performed at the First Affiliated Hospital of Fujian Medical University by the same surgical team who specialize in pancreatic surgery. Patients who did not undergo preoperative MRI or had an MRI at a different hospital were excluded. The final study population $(n=44)$ consisted of 22 patients [ 15 women and 7 men, with a mean age of 53 \pm 13 years (range, 26-79 years)] who developed a PF following PD and 22 control patients [14 women and 8 men, with a mean age of 56 \pm 12 years (range, 35-76 years)] who did not develop a PF. The two groups (fistula and non-fistula group) of patients were carefully matched for perioperative parameters, including age, sex, pancreatic texture, pancreatic duct size at MRI, pancreatic pathology, and type of operation. Demographic, radiology and pathological data are summarized in Table I. The pancreatic texture was evaluated and defined as soft or hard during surgery as described previously $(14,15)$. Soft pancreatic texture was recorded as the level of pancreas elasticity preserved.

Surgical technique and postoperative care. As for the type of surgery performed, conventional PD and pylorus-preserving pancreatoduodenectomy (PPPD) are frequently used for the pancreatic and periampullary cancer at the First Affiliated Hospital of Fujian Medical University. All patients received standard postoperative treatment. A H2 blocker (famotidine; $20 \mathrm{mg}$; trade name, XinFaDing; Shanghai Xinyi Pharmaceutical Co., Ltd., Shanghai, China) was administered intravenously every $12 \mathrm{~h}$ during the non-oral intake period following surgery and octreotide (100 $\mu \mathrm{g}$ Sandostatin; Novartis International AG, Basel, Switzerland) was administered subcutaneously every $8 \mathrm{~h}$ for 5 days. The volume of drained fluids was recorded daily following surgery. The amylase levels in the serum and drainage fluid were measured on the first, third, fifth, seventh and tenth days as described previously $(8,16)$. Serum C-reactive protein and leukocyte count were measured on postoperative day 4 . A plain computed tomography scan was performed to detect potential postoperative complications on day 7 .

According to the definition of the International Study Group on Pancreatic Fistula leak criteria, the postoperative PF was classified into three grades: A, B, and C (17). Tailoring of the treatment strategies was based on this classification.

Pathological analysis. Pancreatic tissue specimens were fixed in $10 \%$ formalin for $8-24 \mathrm{~h}$ at $25^{\circ} \mathrm{C}$, dehydrated in an automatic dehydration processor (Shandon Pathcentre; Thermo Fisher Scientific, Inc., Waltham, MA, USA), and embedded in paraffin, and sectioned serially at a thickness of $4 \mu \mathrm{m}$ following surgical removal. Slides were stained with hematoxylin and eosin (H\&E) for $5-20 \mathrm{~min}$ at $25^{\circ} \mathrm{C}$ and examined under a light microscope (magnification, $\mathrm{x} 200$ ). The severity of acute obstructive pancreatitis was qualitatively assessed by pathological scores as described previously $(18,19)$ : Graded glandular atrophy, scaled from 0-3; intralobular, interlobular and periductal fibrosis, scaled from 0-3; inflammatory cell infiltration, scaled from 0-3. Semi-quantitative pathological scores were obtained by summing up the values for each specimen. Increased pathological scores indicated an increased severity of tissue damage.

MRI protocol and image analysis. MRI examinations were performed on a 3-T imager (Magnetom Verio; Siemens Healthineers, Erlangen, Germany) and a 32-channel phased-array coil. The routine abdominal MRI protocol included fat-suppressed axial and coronal T2-weighted (T2W) turbo spin-echo imaging, T1-weighted dual fast gradient recalled echo sequence (in-phase and out-of-phase sequences) and diffusion-weighted imaging (DWI) with $\mathrm{b}$ values of 0 and $800 \mathrm{~s} / \mathrm{mm}^{2}$. The primary scan parameters are summarized in Table II. Preoperative dynamic MRI pancreatography following intravenous $0.1 \mathrm{mmol} / \mathrm{kg}$ gadolinium contrast treatment with Multihance ${ }^{\circledR}$ gadobenate dimeglumine injection was performed using a volumetric interpolated breath-hold examination with a high-performance phased array sensitivity coding, and the reduction factor was 2 .

ADC maps were automatically generated on a Syngo workstation (Syngo Multimodality Workplace; Siemens Healthineers, Erlangen, Germany) using b-values of 0 and $800 \mathrm{~s} / \mathrm{mm}^{2}$. Regions of interest (ROIs) drawings were performed by consensus between 2 abdominal radiologists with 16 and 8 years of experience, respectively, who were blinded to the clinical history and radiology results. Standard ROI size was 20-45 $\mathrm{mm}^{2}$. A total of 3 repeated measurements on the ADC maps were calculated in the nontumorous pancreatic body and tail, which were residual following PD. Each ROI was manually placed on ADC maps. Special care was taken to avoid the tumor and the primary pancreatic duct, which would have affected ADC values.

On the fat-suppressed T2-weighted (rT2) and unenhanced rT1s, an average signal intensity (SI) of 3 repeated measurements was also calculated from the nontumorous pancreas and ipsilateral muscle. The pancreas-muscle SI ratios on rT1 and rT2 images were calculated as follows: Pancreas-muscle SI ratio $=($ SI pancreas/SI ipsilateral muscle).

Statistical analysis. Data analysis was performed by using SPSS software version 19.0 (IBM Corp., Armonk, NY, USA). Two-tailed $\mathrm{P}<0.05$ was considered to indicate a statistically significant difference. The mean standard deviation (SD) was used to present continuous variables. The Chi-square test or Fisher's exact test was used for comparisons of quantitative parameters, and the Mann-Whitney U test was used for comparisons of qualitative parameters. The ability of ADC values and rT1 to predict the PF development was determined using the receiver-operating characteristic curve (ROC) analysis and 95\% confidence intervals (CI). Point sensitivity and specificity were calculated using ROC curves.

\section{Results}

Patient characteristics. Patient demographic characteristics are summarized in Table I. No differences were observed between the pancreatic fistula group and the non-fistula group in age, 
Table I. Demographic, radiology and pathological data.

\begin{tabular}{|c|c|c|c|}
\hline Characteristics & No fistula group [n=22] (\%) & Fistula group $[\mathrm{n}=22](\%)$ & P-value \\
\hline Age, mean years & $56 \pm 12$ & $53 \pm 13$ & $\mathrm{NS}^{\mathrm{a}}$ \\
\hline Male: Female & $8: 14$ & $7: 15$ & $\mathrm{NS}^{\mathrm{b}}$ \\
\hline \multicolumn{4}{|l|}{ Diagnosis } \\
\hline Pancreas head cancer & $10(45)$ & $11(50)$ & $N S^{b}$ \\
\hline Ampulla of Vater cancer & $9(41)$ & $6(27)$ & $N S^{b}$ \\
\hline Common bile duct cancer & $2(9)$ & $3(14)$ & $\mathrm{NS}^{\mathrm{b}}$ \\
\hline Duodenal cancer & $1(5)$ & $2(9)$ & $\mathrm{NS}^{\mathrm{b}}$ \\
\hline \multicolumn{4}{|l|}{ Type of operation } \\
\hline Conventional pancreaticoduodenectomy & 17 & 16 & $\mathrm{NS}^{\mathrm{b}}$ \\
\hline Pylorus-preserving pancreatoduodenectomy & 5 & 6 & $N S^{b}$ \\
\hline Pancreatic texture at surgery & & & $0.005^{\mathrm{b}}$ \\
\hline Soft & $5(23)$ & $14(64)$ & - \\
\hline Hard & $17(77)$ & $8(36)$ & - \\
\hline Pancreatic duct size on MRI & $3.82 \pm 2.31$ & $2.56 \pm 2.05$ & $0.007^{\mathrm{a}}$ \\
\hline
\end{tabular}

${ }^{a}$ Mann-Whitney U test; ${ }^{b}$ Fisher's exact test or Chi-square test. NS, not significant.

Table II. Primary magnetic resonance imaging parameters.

\begin{tabular}{lcccccc}
\hline Sequence & $\begin{array}{c}\text { TR/TE } \\
(\mathrm{ms})\end{array}$ & $\begin{array}{c}\text { FOV } \\
(\mathrm{mm})\end{array}$ & $\begin{array}{c}\text { Matrix } \\
\text { slice }\end{array}$ & $\begin{array}{c}\text { Thickness/spacing } \\
\text { between slices }\end{array}$ & $\begin{array}{c}\text { Pixel band } \\
\text { width (KHz) }\end{array}$ & $\begin{array}{c}\text { Flip } \\
\text { angle }\left(^{\circ}\right)\end{array}$ \\
\hline $\begin{array}{l}\text { Axial fast spin } \\
\text { echo (T2WI) }\end{array}$ & $2,999 / 79$ & $21 / 38$ & $320 \times 168$ & $5 / 6$ & 240 & 140 \\
$\begin{array}{l}\text { Axial single-shot echo } \\
\text { planar imaging (DWI) }\end{array}$ & $6,000 / 73$ & $21 / 38$ & $128 \times 78$ & $5 / 6$ & 2,441 & 90 \\
$\begin{array}{l}\text { 3D fat-suppressed } \\
\text { gradient-echo (VIBE) }\end{array}$ & $3.9 / 1.4$ & $25 / 38$ & $320 \times 182$ & $3 /-$ & 401 & 9 \\
\hline
\end{tabular}

TR, repetition time; TE, echo time; DWI, diffusion-weighted imaging; FOV, field of view; VIBE, volume interpolated body examination.

sex, pancreatic pathology and type of surgery. Significant differences in pancreatic texture during surgery and pancreatic duct size during MRI examination were identified between the two groups (Table I). In the pancreatic fistula group, 12 patients (55\%) exhibited a grade A fistula, 7 (31\%) exhibited a grade B fistula, and 3 (14\%) exhibited a grade C fistula.

Histopathologic results. Through measuring the grades of severity of obstructive pancreatitis using pathological scores, the score in patients within the fistula group was $5.18 \pm 1.05$, whereas the score of patients within the non-fistula group was $4.32 \pm 1.32$ $(\mathrm{P}=0.021$; Table III). No differences in the areas of glandular atrophy and fibrosis between these two groups were observed. The mononuclear inflammatory infiltrates index of the fistula group was increased compared with the non-fistula group. An increased number of patients in the pancreatic fistula group exhibited a higher grade of severity of acute obstructive pancreatitis.

MRI assessment. The ADC value in the fistula group was $1.14 \pm 0.31 \times 10^{-3} \mathrm{~mm}^{2} / \mathrm{s}$, which was significantly decreased
Table III. Comparison of pathological scores between the fistula and non-fistula groups.

\begin{tabular}{lccc}
\hline Pathological markers & Fistula & No fistula & P-value $^{\mathrm{a}}$ \\
\hline Glandular atrophy & $1.77 \pm 0.81$ & $2.05 \pm 0.79$ & 0.264 \\
Fibrosis & $1.09 \pm 0.68$ & $1.05 \pm 0.72$ & 0.831 \\
Mononuclear & $2.09 \pm 0.68$ & $1.45 \pm 0.59$ & 0.002 \\
inflammatory infiltrates & & & \\
Pathological score & $5.18 \pm 1.05$ & $4.32 \pm 1.32$ & 0.021 \\
\hline
\end{tabular}

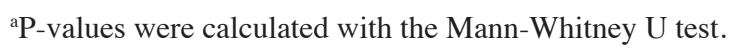

compared with the value in patients in the non-fistula group, which was $1.48 \pm 0.44 \times 10^{-3} \mathrm{~mm}^{2} / \mathrm{s}(\mathrm{P}=0.005)$. The value of the rT1 in patients in the fistula group was $1.71 / 0.25$, which was significantly increased compared with the value of the rT1 of patients in the non-fistula group, which was $1.25 \pm 0.29$ 
Table IV. Signal intensity ratios from fat-suppressed T1WI and T2WI scans, and ADC values between the fistula group and non-fistula groups.

\begin{tabular}{llr}
\hline Measurements & Fistula & No fistula $^{\text {P-value }^{\mathrm{a}}}$ \\
\hline Pancreas-muscle ratio on fat-suppressed T1WI (rT1) & $1.71 \pm 0.25$ & $1.25 \pm 0.29$ \\
Pancreas-muscle ratio on fat-suppressed T2WI (rT2) & $0.72 \pm 0.08$ & $0.62 \pm 0.07$ \\
ADC value & $1.14 \pm 0.31 \times 10^{-3}$ & $1.48 \pm 0.44 \times 10^{-3}$ \\
\hline
\end{tabular}

Data are expressed as mean \pm standard deviation. ${ }^{\text {P }}$-values were calculated with the Mann-Whitney U test. ADC, apparent diffusion coefficient.

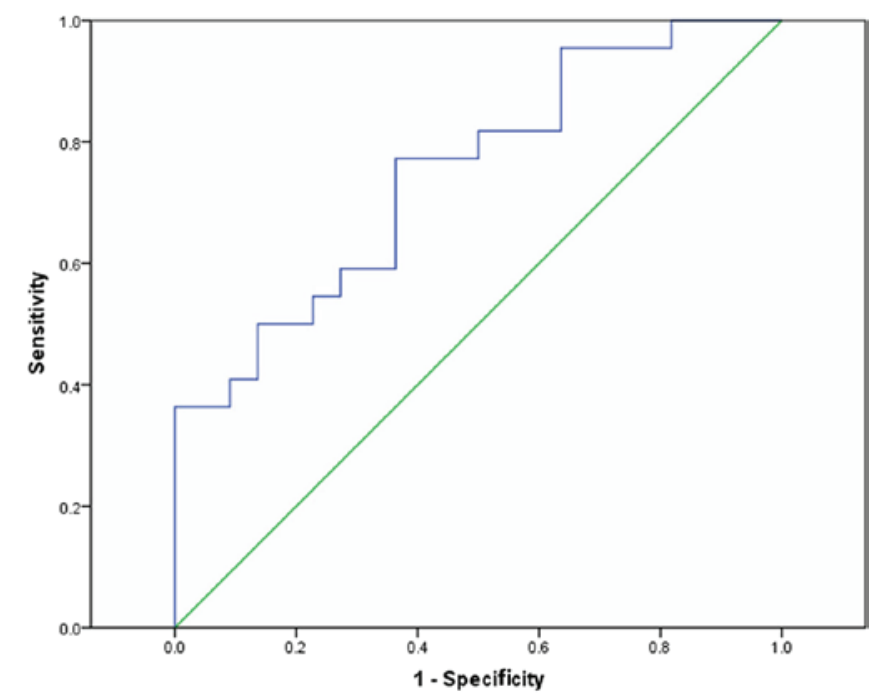

Figure 1. ROC curve of the ADC value. Area under the ROC curve of the ADC value was 0.748 (95\% confidence interval, 0.605-0.891). ROC, receiver operating characteristics; ADC, apparent diffusion coefficient.

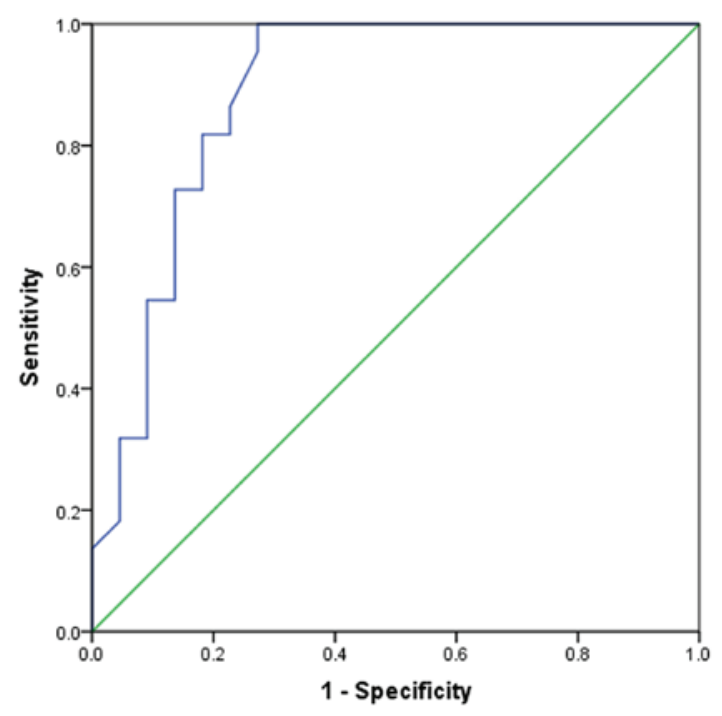

Figure 2. ROC curve of rT1. Area under the ROC curve of rT1 was 0.885 (95\% confidence interval, 0.689-0.959). ROC, receiver operating characteristics.

$(\mathrm{P}=0.0001)$. The value of the $\mathrm{rT} 2$ in patients in the fistula group was $0.72 \pm 0.08$, whereas the value of the rT 2 of patients in the non-fistula group was $0.62 \pm 0.07(\mathrm{P}=0.79)$. No difference in the rT2 values was observed between the two groups (Table IV).
Risk factors of pancreatic fistula. Figs. 1 and 2 demonstrate the diagnostic performance of ADC values and $\mathrm{rT} 1$ parameters for the preoperative prediction of postoperative PF. Based on the ROC curve, the optimal cut-off value of ADC as a criterion for the prediction of PF was $1.29 \times 10^{-3} \mathrm{~mm}^{2} / \mathrm{s}$, which yielded a sensitivity of $77.3 \%$ and a specificity of $63.6 \%$; the area under the curve (AUC) was 0.748 (95\% CI: 0.605-0.891). A cut-off value of rT1 was 0.83 , with the AUC ROC value of 0.885 (95\% CI: 0.689-0.959). Therefore, the diagnostic performance of $\mathrm{rT} 1$ for the preoperative prediction of postoperative PF was better compared with the ADC value.

\section{Discussion}

PF is the primary cause of morbidity following PD, and it has been suggested that the risk for PF depends on a number of variables including age, sex, pancreatic texture and pathology, pancreatic duct size, blood loss, surgery time and surgical techniques (9,20-22). The results of the present study indicated that a significantly increased number of patients with PF exhibited an increased level of mononuclear inflammatory infiltrates and more severe tissue damage of the pancreas due to acute obstructive pancreatitis, as determined by histological examination, compared with patients without PF. Therefore, we hypothesized that pancreatic inflammation and edema were potential risk factors of $\mathrm{PF}$, and the association between acute obstructive pancreatitis and PF was explored using routine abdominal MRI protocol.

ADC values derived from DW-MRI as a quantitative parameter, which demonstrates the Brownian motion of water molecules, may be used to assess all subgroups of acute pancreatitis stratified by the Balthazar classification (23-26). Previous radiology studies have suggested that the ADC value may be used to quantify pancreatic and liver fibrosis (27-29); however, it cannot be used to predict the occurrence of PF (13,30-33). By contrast, Chang et al (34) revealed that ADC values of $\leq 1.3 \times 10^{-3} \mathrm{~mm}^{2} / \mathrm{s}$ may be used to predict the development of PF following PD as a marker of pancreatic fibrosis. In the present study, the routine MRI parameters ADC and rT1 were used to evaluate acute obstructive pancreatitis and determine its predictive ability for PF. These pancreatic MRI imaging protocols may be used to detect and characterize pancreatic and periampullary lesions (13,24,35-37). The present study identified that the optimal cut-off values of ADC and $\mathrm{rT} 1$ as criteria for the prediction of PF was $1.29 \times 10^{-3} \mathrm{~mm}^{2} / \mathrm{s}$ and 0.83 , respectively. These results were consistent with those from previous studies $(9,33,34)$. The diagnostic performance of rT1 (AUC $=0.885)$ for the preoperative prediction of 
postoperative $\mathrm{PF}$ was better compared with the ADC value (AUC=0.748).

In the present study, the pathological score and the mononuclear inflammatory infiltrates of the fistula group, for example the intense degree of edema and glandular inflammation of pancreas, were increased compared with that of the non-fistula group. The likely explanation for the association between acute obstructive pancreatitis and the risk of fistula development was that the pancreatic inflammation and edema will cause anastomosis healing difficulties and the development of leakages. Even a fine needle will cause small pancreatic juice leakages following acupunture.

There were certain limitations in the present study. Firstly, the number of patients was relatively small, and the study was a retrospective analysis. A prospective diagnostic large-scale study should be performed to confirm the results obtained. Secondly, the pathological score, which was calculated in the pancreatic remnant tissue, may not have been an accurate representation of the whole pancreas. Thirdly, postoperative outcomes were not obtained during treatment. Therefore, the gathering of sufficient data was not possible.

The present study demonstrated that MRI parameters including ADC value and rt1 were significantly associated with PF, while rT2 was not. We hypothesize that ADC values and rT1 may predict the occurrence of PF prior to PD, and the prediction ability of rT1 was better compared with ADC values.

The rT1 index may be used as a preoperative predictive tool in PD, similar to the results from a previous study (9). Therefore, these MRI parameters may be applied in predicting the possibility of PF preoperatively. Additional perioperative care should be provided to aid in decreasing the rate of complications when operating on high-risk patients.

In conclusion, ADC values and $\mathrm{rT} 1$ may be a powerful tool for evaluating acute pancreatic obstructive pancreatitis and be useful in predicting the occurrence of PF.

\section{Acknowledgements}

Not applicable.

\section{Funding}

The current study was funded by the Fujian Provincial Department of Science and Technology (grant no. 2016Y0039), the Health and Planning Committee of Fujian Province (grant no. 2016013) and the Health and Planning Committee of Fujian Province (grant no. 2017-CX-27).

\section{Availability of data and materials}

The datasets used and/or analyzed during the current study are available from the corresponding author on reasonable request.

\section{Authors' contributions}

ZS and QC designed the present study. ZS wrote the manuscript and DC, QC and YL revised the manuscript. RY and XL performed the statistical analysis. QC performed imaging analysis. YL and ZS and XL performed MRI scanning procedures. KR and VSL prepared all figures. XL and ZS performed the region of interest analysis and illustrations. DC performed imaging analysis.

\section{Ethics approval and consent to participate}

This retrospective study was approved by the Institutional Review Board of First Affiliated Hospital of Fujian Medical University, and the requirement for informed consent was waived.

\section{Patient consent for publication}

Not applicable.

\section{Competing interests}

The authors declare that they have no competing interests.

\section{References}

1. Freelove R and Walling AD: Pancreatic cancer: Diagnosis and management. Am Fam Physician 73: 485-492, 2006.

2. Balcom JH IV, Rattner DW, Warshaw AL, Chang Y and Fernandez-del Castillo C: Ten-year experience with 733 pancreatic resections: Changing indications, older patients, and decreasing length of hospitalization. Arch Surg 136: 391-398, 2001.

3. Gouma DJ, van Geenen RC, van Gulik TM, de Haan RJ, de Wit LT, Busch OR and Obertop H: Rates of complications and death after pancreaticoduodenectomy: Risk factors and the impact of hospital volume. Ann Surg 232: 786-795, 2000.

4. Cameron JL, Pitt HA, Yeo CJ, Lillemoe KD, Kaufman HS and Coleman J: One hundred and forty-five consecutive pancreaticoduodenectomies without mortality. Ann Surg 217: 430-438, 1993.

5. Aranha GV, Aaron JM, Shoup M and Pickleman J: Current management of pancreatic fistula after pancreaticoduodenectomy. Surgery 140: 561-569, 2006.

6. Bassi C, Butturini G, Molinari E, Mascetta G, Salvia R, Falconi M, Gumbs A and Pederzoli P: Pancreatic fistula rate after pancreatic resection. The importance of definitions. Dig Surg 21: 54-59, 2004.

7. Mathur A, Pitt HA, Marine M, Saxena R, Schmidt CM, Howard TJ, Nakeeb A, Zyromski NJ and Lillemoe KD: Fatty pancreas: A factor in postoperative pancreatic fistula. Ann Surg 246: 1058-1064, 2007.

8. Yoon JH, Lee JM, Lee KB, Kim SW, Kang MJ, Jang JY, Kannengiesser S, Han JK and Choi BI: Pancreatic steatosis and fibrosis: Quantitative assessment with preoperative multiparametric MR imaging. Radiology 279: 140-150, 2016.

9. Kim Z, Kim MJ, Kim JH, Jin SY, Kim YB, Seo D, Choi D, Hur KY, Kim JJ, Lee MH and Moon C: Prediction of post-operative pancreatic fistula in pancreaticoduodenectomy patients using pre-operative MRI: A pilot study. HPB (Oxford) 11: 215-221, 2009.

10. Scialpi M, Cagini L, Pierotti L, De Santis F, Pusiol T, Piscioli I, Magli M, D'Andrea A, Brunese L and Rotondo A: Detection of small $(\leq 2 \mathrm{~cm})$ pancreatic adenocarcinoma and surrounding parenchyma: Correlations between enhancement patterns at triphasic MDCT and histologic features. BMC Gastroenterol 14: $16,2014$.

11. Fukumura Y, Kumasaka T, Mitani K, Karita K and Suda K: Expression of transforming growth factor beta1, beta2, and beta3 in chronic, cancer-associated, obstructive pancreatitis. Arch Pathol Lab Med 130: 356-361, 2006.

12. Kamisawa T, Takuma K, Anjiki H, Egawa N, Hata T, Kurata M, Honda G, Tsuruta K, Suzuki M, Kamata N and Sasaki T: Differentiation of autoimmune pancreatitis from pancreatic cancer by diffusion-weighted MRI. Am J Gastroenterol 105: 1870-1875, 2010.

13. Muraoka N, Uematsu H, Kimura H, Imamura Y, Fujiwara Y, Murakami M, Yamaguchi A and Itoh H: Apparent diffusion coefficient in pancreatic cancer: Characterization and histopathological correlations. J Magn Reson Imaging 27: 1302-1308, 2008. 
14. Rosso E, Casnedi S, Pessaux P, Oussoultzoglou E, Panaro F, Mahfud M, Jaeck D and Bachellier P: The role of 'fatty pancreas' and of BMI in the occurrence of pancreatic fistula after pancreaticoduodenectomy. J Gastrointest Surg 13: 1845-1851, 2009.

15. Lee SE, Jang JY, Lim CS, Kang MJ, Kim SH, Kim MA and Kim SW: Measurement of pancreatic fat by magnetic resonance imaging: Predicting the occurrence of pancreatic fistula after pancreatoduodenectomy. Ann Surg 251: 932-936, 2010.

16. Molinari E, Bassi C, Salvia R, Butturini G, Crippa S, Talamini G, Falconi $\mathrm{M}$ and Pederzoli P: Amylase value in drains after pancreatic resection as predictive factor of postoperative pancreatic fistula: Results of a prospective study in 137 patients. Ann Surg 246: 281-287, 2007.

17. Bassi C, Dervenis C, Butturini G, Fingerhut A, Yeo C, Izbicki J, Neoptolemos J, Sarr M, Traverso W and Buchler M; International Study Group on Pancreatic Fistula Definition: Postoperative pancreatic fistula: An international study group (ISGPF) definition. Surgery 138: 8-13, 2005.

18. Puig-Diví V, Molero X, Vaquero E, Salas A, Guarner F and Malagelada J: Ethanol feeding aggravates morphological and biochemical parameters in experimental chronic pancreatitis. Digestion 60: 166-174, 1999.

19. Puig-Diví V, Molero X, Salas A, Guarner F, Guarner L and Malagelada JR: Induction of chronic pancreatic disease by trinitrobenzene sulfonic acid infusion into rat pancreatic ducts. Pancreas 13: 417-424, 1996.

20. Butturini G, Marcucci S, Molinari E, Mascetta G, Landoni L, Crippa S and Bassi C: Complications after pancreaticoduodenectomy: The problem of current definitions. J Hepatobiliary Pancreat Surg 13: 207-211, 2006.

21. Tajima Y, Kuroki T, Tsuneoka N, Adachi T, Kosaka T, Okamoto T, Takatsuki M, Eguchi S and Kanematsu T: Anatomy-specific pancreatic stump management to reduce the risk of pancreatic fistula after pancreatic head resection. World J Surg 33: 2166-2176, 2009

22. Sanjay P, Fawzi A, Fulke JL, Kulli C, Tait IS, Zealley IA and Polignano FM: Late post pancreatectomy haemorrhage. Risk factors and modern management. JOP 11: 220-225, 2010.

23. Yencilek E, Telli S, Tekesin K, Ozgür A, Cakır O, Türkoğlu O, Meric K and Simsek M: The efficacy of diffusion weighted imaging for detection of acute pancreatitis and comparison of subgroups according to Balthazar classification. Turk J Gastroenterol 25: 553-557, 2014.

24. Akisik MF, Aisen AM, Sandrasegaran K, Jennings SG, Lin C, Sherman S, Lin JA and Rydberg M: Assessment of chronic pancreatitis: Utility of diffusion-weighted MR imaging with secretin enhancement. Radiology 250: 103-109, 2009.

25. Shinya S, Sasaki T, Nakagawa Y, Guiquing Z, Yamamoto F and Yamashita Y: Acute pancreatitis successfully diagnosed by diffusion-weighted imaging: A case report. World J Gastroenterol 14: 5478-5480, 2008.

26. Frozanpor F, Loizou L, Ansorge C, Segersvärd R, Lundell L and Albiin N: Preoperative pancreas CT/MRI characteristics predict fistula rate after pancreaticoduodenectomy. World J Surg 36: $1858-1865,2012$
27. Lewin M, Poujol-Robert A, Boëlle PY, Wendum D, Lasnier E, Viallon M, Guéchot J, Hoeffel C, Arrivé L, Tubiana JM and Poupon R: Diffusion-weighted magnetic resonance imaging for the assessment of fibrosis in chronic hepatitis C. Hepatology 46: 658-665, 2007.

28. Yoshikawa T, Kawamitsu H, Mitchell DG, Ohno Y, Ku Y, Seo Y, Fujii $M$ and Sugimura K: ADC measurement of abdominal organs and lesions using parallel imaging technique. AJR Am J Roentgenol 187: 1521-1530, 2006.

29. Patel J, Sigmund EE, Rusinek H, Oei M, Babb JS and Taouli B: Diagnosis of cirrhosis with intravoxel incoherent motion diffusion MRI and dynamic contrast-enhanced MRI alone and in combination: Preliminary experience. J Magn Reson Imaging 31: 589-600, 2010

30. Kah Heng CA, Salleh I, San TS, Ying F and Su-Ming T: Pancreatic fistula after distal pancreatectomy: Incidence, risk factors and management. ANZ J Surg 80: 619-623, 2010.

31. Watanabe H, Kanematsu M, Tanaka K, Osada S, Tomita H, Hara A, Goshima S, Kondo H, Kawada H, Noda Y, et al: Fibrosis and postoperative fistula of the pancreas: Correlation with MR imaging findings-preliminary results. Radiology 270: 791-799, 2014.

32. Jimenez RE and Hawkins WG: Emerging strategies to prevent the development of pancreatic fistula after distal pancreatectomy. Surgery 152 (3 Suppl 1): S64-S70, 2012.

33. Harada N, Ishizawa T, Inoue Y, Aoki T, Sakamoto Y, Hasegawa K, Sugawara Y, Tanaka M, Fukayama M and Kokudo N: Acoustic radiation force impulse imaging of the pancreas for estimation of pathologic fibrosis and risk of postoperative pancreatic fistula. J Am Coll Surg 219: 887-894.e5, 2014.

34. Chang YR, Kang JS, Jang JY, Jung WH, Kang MJ, Lee KB and Kim SW: Prediction of pancreatic fistula after distal pancreatectomy based on cross-sectional images. World J Surg 41: 1610-1617, 2017.

35. Wiggermann $P$, Grützmann R, Weissenböck A, Kamusella $P$, Dittert DD and Stroszczynski C: Apparent diffusion coefficient measurements of the pancreas, pancreas carcinoma, and mass-forming focal pancreatitis. Acta Radiol 53: 135-139, 2012.

36. Gallix BP, Bret PM, Atri M, Lecesne R and Reinhold C: Comparison of qualitative and quantitative measurements on unenhanced T1-weighted fat saturation MR images in predicting pancreatic pathology. J Magn Reson Imaging 21: 583-589, 2005

37. Barral M, Sebbag-Sfez D, Hoeffel C, Chaput U, Dohan A, Eveno C, Boudiaf M and Soyer P: Characterization of focal pancreatic lesions using normalized apparent diffusion coefficient at 1.5-Tesla: Preliminary experience. Diagn Interv Imaging 94: 619-627, 2013. 